\title{
Wind Turbine Blade Composites Assessment Using Non-Contact Ultrasound Method
}

\author{
Rozina Steigmann, Nicoleta Iftimie, Adriana Savin, and Roman Sturm
}

\begin{abstract}
Wind turbine blades (WTB) are one of the most damageable components of a wind turbine system and due to their importance must be tested during the fabrication and before installing. WTB are produced from composite materials with polymeric matrices, most of the stages of producing are manufacturing and can result in different types of defects. Therefore, non-destructive testing (NDT) techniques that provide surface and internal information of the blade are required. In this paper, ultrasonic testing using noncontact transducers for the control of composites type Glass Fiber Reinforced Plastics (GFRP) with orthophthalic polyester resins matrix is presented in order to prove its capabilities for such applications. The mechanical properties determined by above mentioned method are confirmed by destructive tests using Dynamic Mechanical Analysis.
\end{abstract}

Index Terms - Wind turbine blade, GFRP, nondestructive testing, non-contact ultrasonic method.

\section{INTRODUCTION}

Historically, wind machines were used for grinding grains in Persia as early as 200BC.

Wind turbines have been in use since 1941 when the world's first megawatt -size wind turbine was connected to the local electrical distribution system in Vermont USA.

Promoting clean energy technologies, environmental protection measures and reduction of greenhouse gas has become a priority in the energy industry. The production of green energy has considerable increased in the last decade. While overall primary energy supply from renewables in has grown with $30 \%$ from 2004 to 2013, by 2014, renewables supplied approximately $19 \%$ of the world's final energy consumption [1]. Production of wind power saw a similar increase moving from a total installed capacity of $48 \mathrm{GW}$ in 2004 to $318 \mathrm{GW}$ in 2014. The scenario for 2020 foresees a figure of $230 \mathrm{GW}$ (of which $40 \mathrm{GW}$ offshore) producing 581 TWh of electricity, meeting $15.7 \%$ of electricity consumption EU electricity consumption for 2020 was projected to be 3,689.5 TWh [2].

These needs lead to an efficiency in construction and maintenance of wind turbine. While wind turbines on duty are relied to work $90 \%$ of the time, many structural flows are still

Manuscript received April 29, 2015; revised October 10, 2015.

R. Steigmann is with the Nondestructive Testing Department, National Institute of Research and Development for Technical Physics, Iasi, Romania. He is also with the Faculty of Physics, University Al. I. Cuza, Iasi, Romania (e-mail: steigmann@phys-iasi.ro).

A. Savin and N. Iftimie are with the Nondestructive Testing Department, National Institute of Research and Development for Technical Physics, Iasi, Romania.

R. Sturm is with the Faculty of Mechanical Engineering, University of Ljubljana, Ljubljana, Slovenia. encountered, particularly with the blades. Wind turbine blades (WTB) are one of the most frequently damaged components, can develop cracks at the edges, near the hub or at the tips.

The challenge for the producers is to find materials with operational parameters and conditions leading to the following requirements [3]: focused on stiffness, density, and long-time fatigue; high material stiffness is needed to maintain optimal aerodynamic performance; low density is needed to reduce gravity forces; long-fatigue life is needed to reduce material degradation. Therefore, most of the wind turbine producers combine the use of glass fiber reinforced plastics (GFRP) [4], [5] and carbon fiber reinforced plastics (CFRP) [6], [7] as composites in WTB fabrication (Fig. 1).

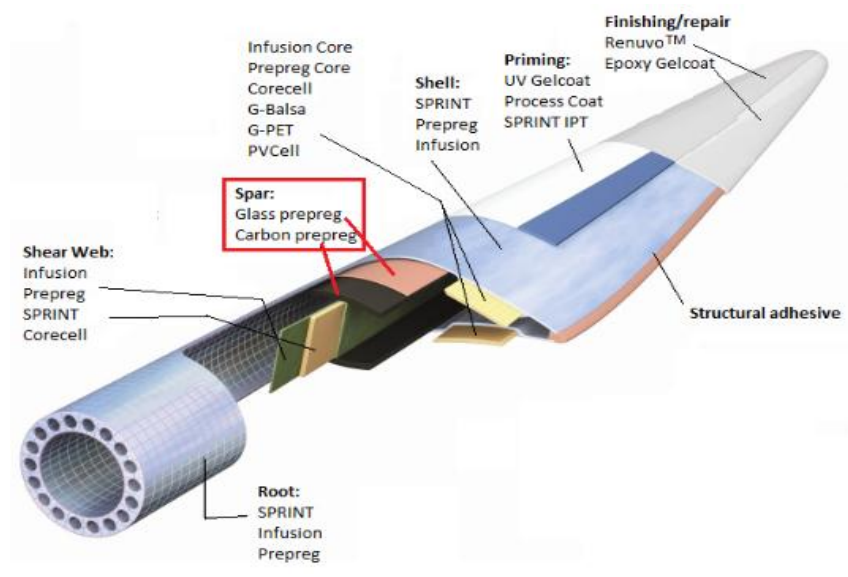

Fig. 1. Wind turbine blade [8].

But composites exhibit few damage mechanisms: fiber failure, fiber/matrix debonding, matrix cracking, delaminations, dry zones and voids, porosities due to poor curing [9].

Thus, these materials must be inspected, as nondestructively as is possible in order to avoid compromising their quality. Ultrasonic, shearography, thermography and X-ray CT techniques are usually used for the inspection of wind turbine blades [10]-[14]. One of the most suitable methods for nondestructive evaluation of GFRP is ultrasound [15]. While ultrasound and its applications have grown phenomenally in the recent years, the mode by which it is transmitted in a given test medium is severely limited by physical contact between the transducer and the test medium by a liquid gel.

In this paper, it presented a method for nondestructive evaluation of the composite materials used in manufacturing of WTB, involving air-coupled ultrasound transducers. The method presents the advantages of not using contact fluid and not requiring access from the both sides of the samples. 


\section{STUDIED SAMPLES AND EXPERIMENTAL SET-UP}

Typically, for wind turbines blades skins are the composites GFRP. GFRP plates can be reinforced with a variable number of layers. We have studied GFRP having as reinforcement 6 sheets of ravings with $250 \pm 50 \mathrm{gm}^{-2}$ density of glass fibers and matrix from different types of unsaturated orthophthalic polyester resins (Fig. 2(a)). The GFRP samples taken into study, made by Helios, Slovenia, have the matrix made by COLPOLY Slovenia. From raw specimens, specimens were cropped in order to make mechanical tests (Fig. 2(b)-(c)).

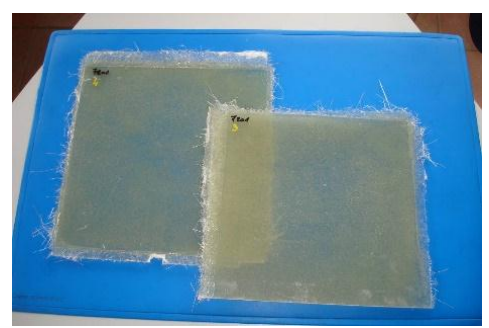

(a)

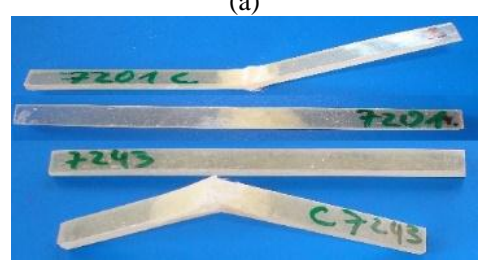

(b)

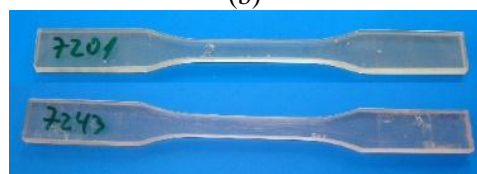

(c)

Fig. 2. Studied samples: a) raw specimens; b) GFRP studied samples; c) dog-bone shapes from resin.

The features of the samples are presented in Table I.

TABLE 1: STUDIED GFRP SAMPLES

\begin{tabular}{lllllll}
\hline \hline No. & $\begin{array}{l}\text { Sample } \\
\text { name }\end{array}$ & Matrix & $\begin{array}{l}\text { No. of } \\
\text { layers }\end{array}$ & $\begin{array}{l}\text { Fiber value } \\
\text { ratio }\end{array}$ & $\begin{array}{l}\text { Density } \\
{[\mathrm{kg} \mathrm{m}-3]}\end{array}$ & Observation \\
\hline 1 & $7201-61$ & $\begin{array}{l}\text { Colpoly } \\
7201\end{array}$ & 6 & $0.6 \pm 0.05$ & $1550 \pm 20$ & $\begin{array}{l}\text { Medium } \\
\text { reactivity resin }\end{array}$ \\
2 & $7243-61$ & $\begin{array}{l}\text { Colpoly } \\
7243\end{array}$ & 6 & $0.6 \pm 0.05$ & $1410 \pm 20$ & $\begin{array}{l}\text { Preaccelerated } \\
\text { thixotropic }\end{array}$ \\
\hline \hline
\end{tabular}

The dog bone samples have the dimensions specified in Fig. 3. The static tests were carried out both for GFRP and the resin matrix using Instron E 8801, having hydraulic fixture for composites (Fig. 4). Samples having dimensions of $50 \times 10 \times 3.9 \mathrm{~mm}^{3}$ were cropped for dynamic tests in order to determinate complex elastic modulus, shear modulus using a Dynamic Mechanical Analyzer DMA 242 Netzsch Germany with 3 points bending fixture (Fig. 5).

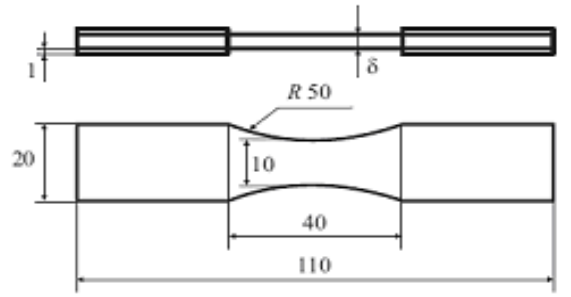

Fig. 3. Shape and dimensions of dog bone specimens for fatigue tests.

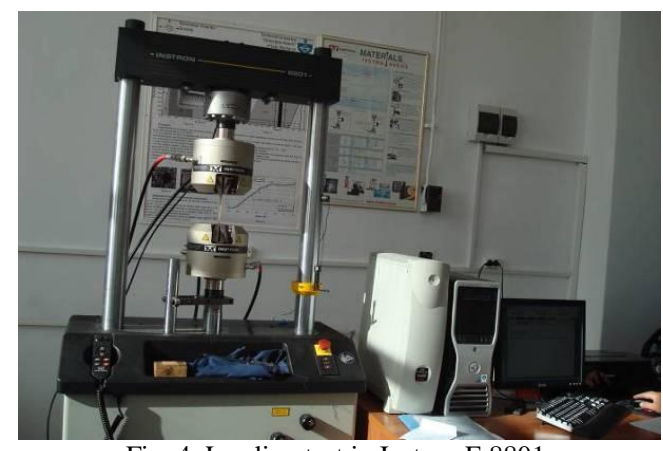

Fig. 4. Loading test in Instron E 8801.

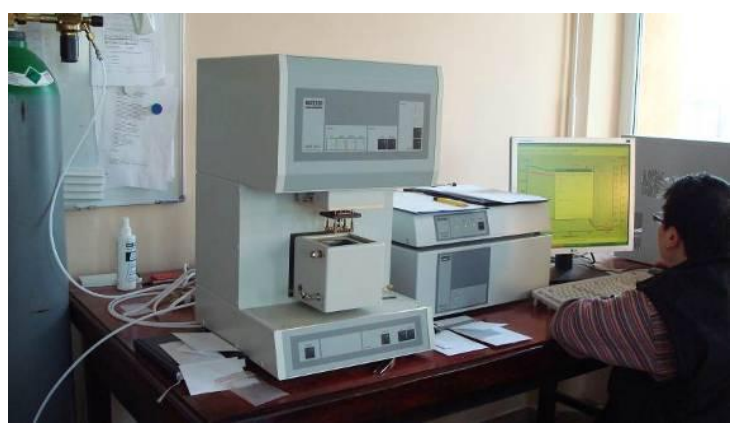

(a)

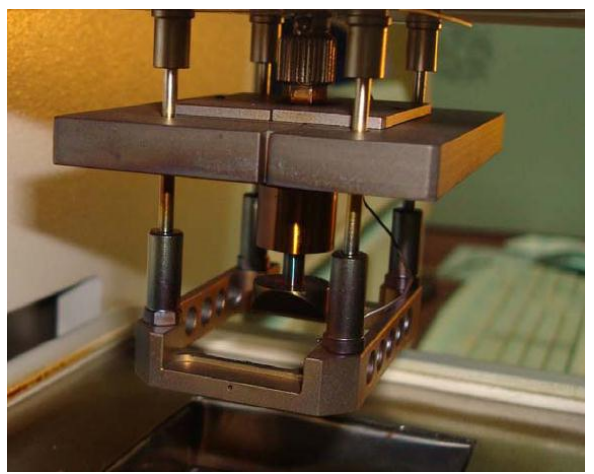

(b)

Fig. 5. Dynamic mechanical analyzer: a) the equipment; b) 3 points bending test fixture.

The mechanical characteristics, glass transition temperature and activation energy [16] determined using DMA 242 C and Instron E 8801 are presented Table II.

TABLE II: MECHANICAL PROPERTIES DETERMINED BY DMA

\begin{tabular}{llllll}
\hline \hline Composite & $\begin{array}{l}E x=E y \\
{[\mathrm{GPa}]}\end{array}$ & $\begin{array}{l}E z \\
{[\mathrm{GPa}]}\end{array}$ & $v_{x y}$ & $\begin{array}{l}T g \\
{[\mathrm{OC}]}\end{array}$ & $\begin{array}{l}\text { Activation } \\
\text { energy } \\
{[\mathrm{kJ} / \mathrm{mol}]}\end{array}$ \\
\hline 7201 & 9.1 & 8.2 & 0.2 & 82.9 & 149.6 \\
7243 & 8.4 & 8.2 & 0.2 & 105.7 & 296.4 \\
\hline \hline
\end{tabular}

$E z, v x z$ and $G x z$ were determined from the measurement of propagation speed of the ultrasound along the direction $Z$ (according to Fig. 6), the other mechanical properties being determined from the propagation speed of the Lamb waves, the fundamental modes $S_{0}$ and $A_{0}$, where $E_{z}$ is Young modulus, $G_{x z}$ is shear modulus and $v_{x z}$ Poisson ratio can be determined by measuring the velocity of longitudinal waves and transversal waves that propagate along $z$ direction [17].

In Fig. 7, it can be observed the variation of Young modulus for each type of composites. Five specimens were cropped from each studied samples. For further investigation with noncontact ultrasound, the first composite (sample 7201) was chosen, having higher Young modulus and density. 


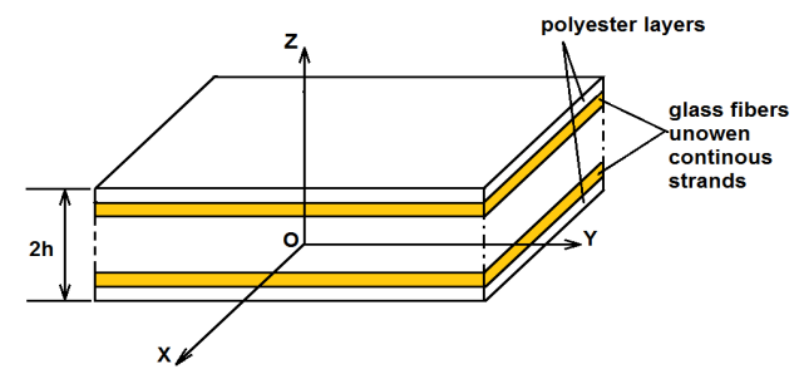

Fig. 6. The composite layout.

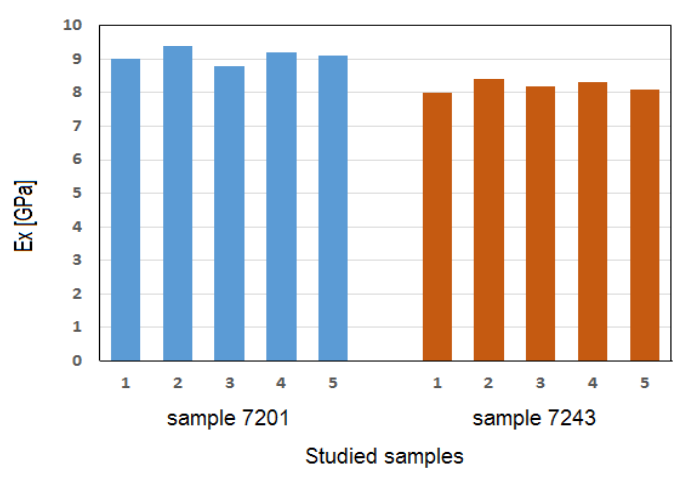

Fig. 7. Young modulus for studied samples.

In Fig. 8, it presented the results obtained from DMA for 7201-61 sample. The evaluation of elastic properties of GFRP composites was nondestructively made using an ultrasound method with air coupled transducers type NGC 100D25 Ultran Group USA, having the central frequency of $100 \mathrm{kHz}$.

The excitation with rectangular impulses of the emission transducer was made by a Pulser-Receiver PR 5073, Panametrics USA.

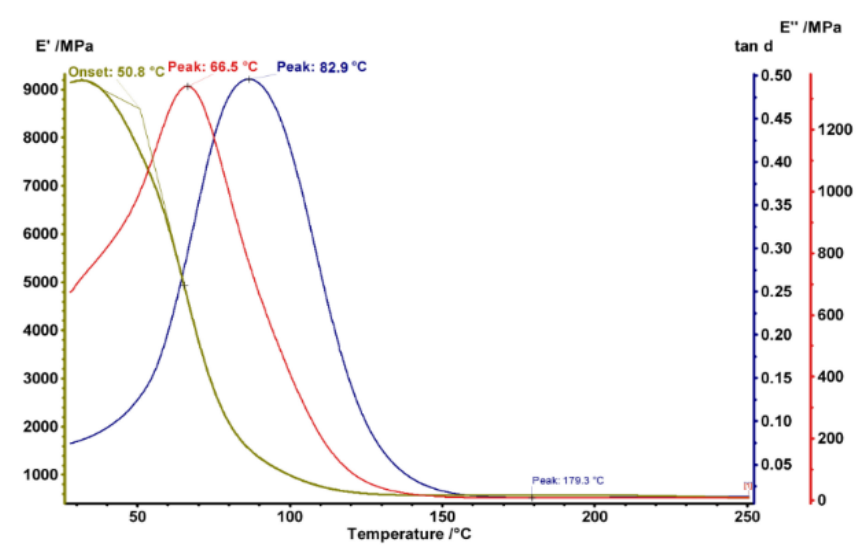

Fig. 8. DMA results for 7201-61 sample.

The received signal is pre-amplified with a preamplifier Ultrasonic Preamp - Panametrics USA and convenient processed in pulser-receiver. The digitization of the signals is made with a digital oscilloscope Wave Runner 64Xi - LeCroy USA coupled with a PC. In order to obtain the changes of the modes in the central incidence point, the emission and reception transducers were mounted so that they formed $11^{0}$ angles with the normal at composite surface, in composite being generated both longitudinal and transversal ultrasound wave which form together the Lamb waves. The initial distance between the central incidence point and the reception point has been varied between $80 \mathrm{~mm}$ and $180 \mathrm{~mm}$, in $5 \mathrm{~mm}$ steps, the received wave shape being saved for each situation. The experimental set-up is presented in Fig. 9.

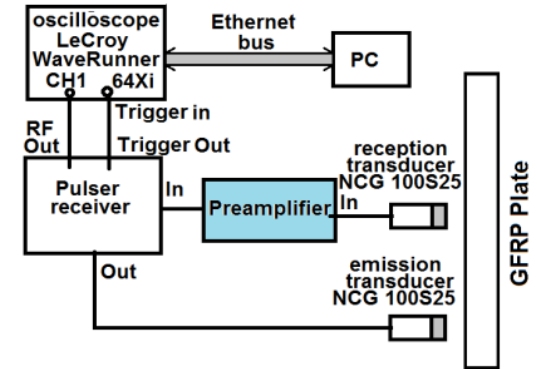

(a)

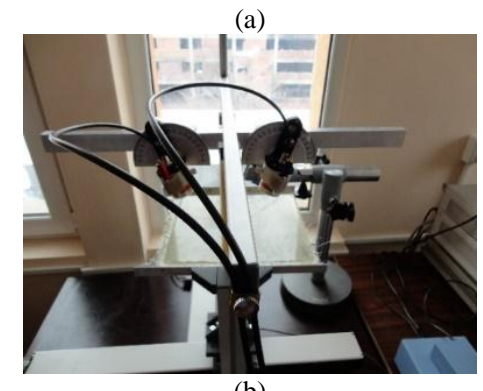

(b)

Fig. 9. Experimental set-up: a) scheme; b) transducers layout.

\section{REsults}

The collection of signals received for different distances between the central incidence point and the reception one was organized into a bi-dimensional matrix, a linear vector corresponding to each signal. In Fig. 10, it presented several signal registered for the studied sample, the distance where the signal has been received being marked on graph. In Fig. 11(a) is presented the signal received by the reception transducer at the examination of 7201 composite, where the modes A0, S0 and SH0 can be distinguished. For a more reliable identification, the amplitude spectrum has been traced and presented in Fig. 11(b). In Fig. 11(c), it presented the corresponding spectrogram.

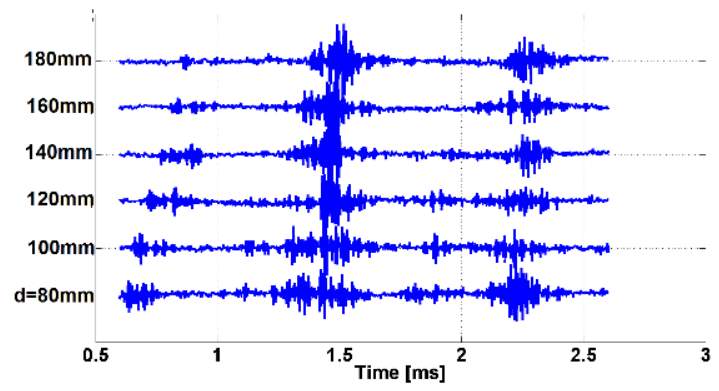

Fig. 10. The Lamb waves modes received to different distances between the incidence point and reception point for the studied sample.

The signal is presented in time-domain (Fig. 11(a)), frequency domain (Fig. 11(b)) and as spectrogram (Fig. 11(c)).

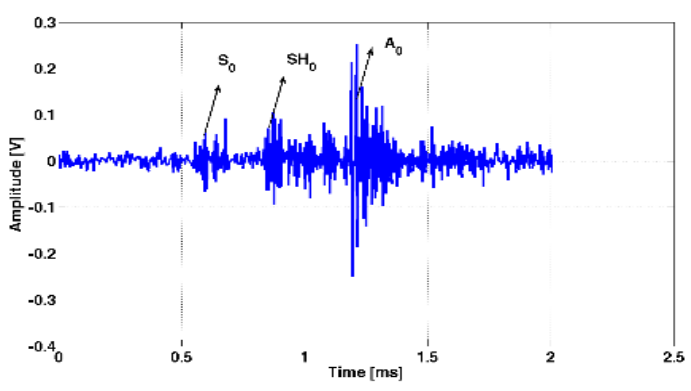

(a) 


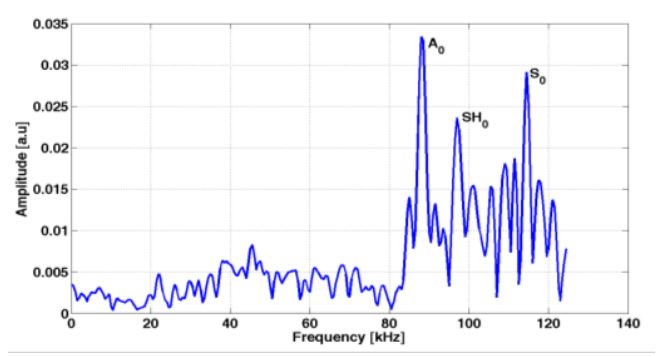

(b)

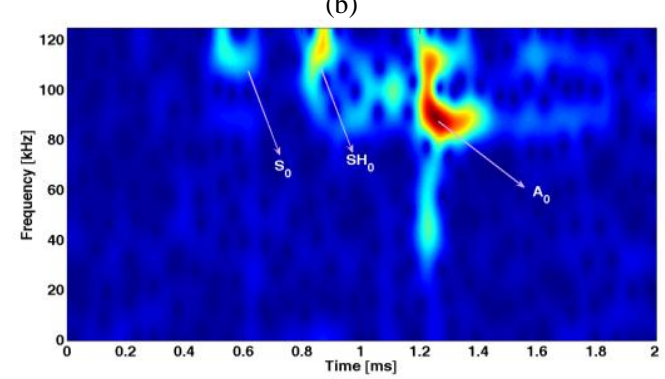

(c)

Fig. 11. The experimental results: a) The signal received by the reception transducer at the examination of 72011 composite; b) the amplitude spectrum; c) the spectrogram.

\section{CONCLUSIONS}

GFRP composites are used in construction of wind turbine blades due their good operational, mechanical and physical parameters and also due their low cost with respect to carbon fibers. The performance and behavior characteristics of nearly all in-service composite structures can be affected by degradation resulting from sustained use as well as from exposure to severe environmental conditions or damage due to external conditions such as impacts, loading abrasion, operator abuse or neglect. These factors can have serious consequence on the structures relative to safety, costs and operational capability. WTB are one of the most damageable components of WT systems, even before installing. Air coupled ultrasound transducers in pitch-catch configuration allows the generation and reception of Lamb waves generated in GFRP plates without using coupling and are used for analysis of GFRP composites with two types of matrix. The elements of elastic matrix of GFRP are correlated directly with the phase velocity of different symmetric and antisymmetric modes of Lamb waves leading to determination of the elastic modulus, share modulus and Poisson coefficients in order to choose the characteristics that promote one of composite samples as good candidate for using in WTB manufacturing. These results are in good concordance with those obtained by classical destructive methods as dynamic mechanical analysis and loading tests. The results showed good mechanical results for GFRP composite with reinforcement from 6 sheets of glass fibers ravings, having $250 \pm 50 \mathrm{gm}-2$ density of fibers and matrix COLPOLY 7201 medium reactivity resin produced in two steps.

Comparatively to conventional ultrasound method, non-contact ultrasound has limit imposed by the complexity of material shape and size. However, it can be used in WTB investigation due to large plane surface of the blade.

\section{ACKNOWLEDGMENT}

The manuscript was written through contributions of all authors. All authors have given approval to the final version of the manuscript. All authors contributed equally.

This paper is partially supported by the Romanian Ministry of Education MEN - UEFISCDI, project no. PN-II-PCCA-2013-4-0656 Partnership, project no. PN-II-ID-PCE-2012-4-0437 Ideas, Nucleus program, Contract no. 09-43-01-04 and by the strategic grant POSDRU/159/1.5/S/137750, co-financed by the ESF within the Sectorial Operational Program Human Resources Development 2007-2013.

\section{REFERENCES}

[1] Pure Power - Wind Energy Targets for 2020 and 2030, EWEA, 2011.

[2] Renewable Energy Policy Network for the 21st Century, REN21 Report, 2014.

[3] M. F. Ashby, Materials Selection in Mechanical Design, $3^{\text {rd }}$ ed., Pergamon Press, 2002.

[4] R. J. Bussolari, "Fibreglass composite blades for the 4MW-WTS-4 wind turbine," NASA-Lewis Research Center Large Horizontal-Axis Wind Turbines, pp. 259-266, 1983.

[5] P. Brøndsted, H. Lilholt, and A. Lystrup, "Composite materials for wind power turbine blades," Annu. Rev. Mater. Res., vol. 35, pp. 505-538, 2005.

[6] P. Hogg, "Wind turbine blade materials," Supergen Wind Phase 1 Final Assembly, Loughborough, March 2010.

[7] R. Stewart, "Wind turbine blade production - new products keep pace as scale increases," Reinforced Plastics, pp. 19-25, 2012.

[8] Gurit. [Online]. Available: http://gurit.fangle.co.uk/

[9] P. Morgan, Carbon Fibers and Their Composites, Taylor \& Francis, NY, 2005

[10] E. Bayraktar, S. D. Antolovich, and C. Bathias, "New developments in non-destructive controls of the composite materials and applications in manufacturing engineering," Journal of Materials Processing Technology, vol. 206, no. 1, pp. 30-44, 2008.

[11] R. Raišutis, E. Jasiūnienè, and E. Žukauskas, "Ultrasonic NDT of wind turbine blades using guided waves," Ultragarsas (ultrasound), vol. 63, no. 1, pp. 7-11, 2008.

[12] I. Amenabar, A. Mendikute, A. López-Arraiza, M. Lizaranzu, and J. Aurrekoetxea, "Comparison and analysis of non-destructive testing techniques suitable for delamination inspection in wind turbine blades," Compos B Eng, vol. 42, no. 5, pp. 1298-305, 2011.

[13] M. A. Drewry and G. A. Georgiou, "A review of NDT techniques for wind turbine blades," INSIGHT, vol. 49, pp. 137-141, 2007.

[14] W. T. Estler, L. Edmundson, G. N. Peggs, and N. Parker, "Large-scale metrology - an update," Ann CIRP, vol. 51, pp. 587-609, 2002.

[15] C. Scarponi and G. Briotti, "Ultrasonic technique for the evaluation of delaminations on CFRP, GFRP, KFRP," Composites Part B: Engineering, vol. 31, no. 3, pp. 237-243, 2000.

[16] K. P. Menard, Dynamic Mechanical Analysis - A Practical Introduction, CRC Press, 1999.

[17] J. Krautkramer and H. Krautkramer, Ultrasonic Testing of Materials, 4th ed., Springer Verlag, 1990.

Rozina Steigmann has received the master degree in 1999. He has been a Ph.D. Student in 2012, at the Faculty of Physics, University Alexandru Ioan Cuza, Iasi, Romania. Now he is also with the Nondestructive Testing Department, National Institute of Research and Development for Technical Physics, Iasi, Romania. 